\title{
Motivos da prática esportiva de acordo com o nível de com- petência percebida na idade adulta: um estudo piloto
}

\author{
Ana Pavón Lores ${ }^{1}$, Juan Antonio Moreno Murcia ${ }^{2}$, Estélio H. M. Dantas ${ }^{3}$ \\ ${ }^{1}$ Unidad de Investigación en Educación Física y Deportes, Espanha. \\ ${ }^{2}$ Universidad de Murcia, Facultad de Ciencias del Deporte, Murcia, Espanha. \\ ${ }^{3}$ Universidade castelo Branco, Rio de Janeiro, RJ, Brasil.
}

\author{
Lores, A.; Murcia, J.; Dantas, E.; Motivos da \\ prática esportiva de acordo com o nível de \\ competência percebida na idade adulta: um \\ estudo piloto. Motricidade 3(4): 7-21
}

\section{Resumo}

Levando em conta que os interesses evoluem ao longo das diferentes idades, e sua inquestionável influência sobre o nível de prática esportiva na idade adulta, decidimos realizar uma análise do que motiva os universitários à prática das atividades físico-esportivas, considerando diversos fatores como gênero, idade, nível de prática e gosto pelo esporte. Com este fim, desenvolvemos o Questionário dos Interesses nas Atividades Físico-Esportivas e as Motivações que levam à Prática das mesmas numa amostra de 1512 estudantes universitários. Assim, os praticantes de atividades físico-esportivas explicam a participação neste tipo de atividades, por motivos vinculados à manutenção da forma física e aos benefícios que o exercício físico traz à saúde; aqueles que nunca praticaram argumentam que a prática físico-esportiva tira tempo de estudo e demonstram na falta de interesse, e os alunos que manifestam ter praticado algum esporte anteriormente, mas que deixaram de praticá-lo, indicam a falta de tempo livre e o nível de exigência dos estudos como razões para explicar o seu comportamento físico-esportivo. Se trata de um estudo preliminar e futuramente o questinário deverá ser validado através de análise estatística.

Palavras-chave: Motivação, atividade físico-esportiva, esporte, Universidade, Educação Física.

\section{Abstract}

Reasons for physical practice according to the perceived competence level: a pilot study

Taking into account that the interests evolve along the different ages, and their unquestionable influence on the level of physical activity in adults, we have decided to carry out an analysis of the motivations of the university students toward the physical and sport activities, assisting to diverse factors like the gender, the age, the practice level and the pleasure for the sport. Therefore, we have developed the Questionnaire dealing with motivations and interests in sport and physical activities on a sample of 1512 university students. So, the practitioners of physical and sport activities will explain their participation in this activities, for reasons linked to keep fit and to the benefits of the exercise for the health; the non practitioners base their behavior in that the physical and sport practice removes time for the study and in the lack of interest, and the students to have practiced sport previously, but that they have stopped to practice it at the moment, they point out the lack of spare time and the level of requirement of their studies like reasons to explain their sport behavior. If it deals with a preliminary study and future the questinário will have to be validated through statistics analysis.

Keywords: Motivation, physical activity, sport, University, Physical Education. 


\section{Introdução}

A magnitude social do fenômeno esportivo junto às numerosas pesquisas, em que se demonstra a correlação positiva entre a conservação de estilos de vida ativos e estados de saúde e bem-estar, constituem o ponto de partida para o crescente interesse no desenvolvimento dos aspectos relativos ao conhecimento e à compreensão dos fatores que determinam que os indivíduos mantenham estilos de vida ativos ${ }^{1,13}$.

Assim, os indivíduos com atitudes positivas com relação ao exercício, geralmente têm comportamentos de exercício físico mais intensos e mais frequentes do que as pessoas que têm menos atitudes positivas com relação ao mesmo. Tendência esta que foi corroborada por pesquisas posteriores $^{36,24}$, em que se constatava a influência das experiências com relação à participação em atividades físicas durante a infância e a adolescência e o nível de atividade física dos adultos, ou aquelas que confirmam a existência de uma correlação positiva significativa entre a atividade física durante a infância e a adolescência, destacando que a inatividade física mostra uma melhor possibilidade de previsão do que a própria atividade.

Além do mais, a natureza e o tipo de programas de atividade física selecionados podem ter influência no tipo de atividade que os alunos escolhem para participar depois da formatura. Portanto, não devemos esquecer de que as pessoas ativas o são ao longo da vida e as sedentárias se mantêm como tais independentemente das circunstâncias, existindo uma grande influência da própria estória de atividade física do indivíduo para determinar o nível atual de atividade física ${ }^{1,30}$, já que os universitários são conscientes das contribuições positivas que a participação de atividades físicas de forma regular têm sobre um estilo de vida saudável e ativo ${ }^{32,31}$.

Por isso, se partirmos das conclusões de Masachs et al. que dizem que os motivos se mantêm ou se modificam à medida que se pratica o exercício ${ }^{16}$ há um período de tempo, será interessante observar como evoluem os interesses na atividade esportiva ao longo das diferentes idades, pela sua inquestionável influência sobre o nível de prática esportiva na idade adulta. Daí a importância de analisar quais serão os interesses e as motivações que levam os estudantes universitários à prática ou ao abandono das atividades físicas, levando em conta, principalmente, os importantes benefícios que trazem à saúde física e mental.

Assim, o objetivo desta pesquisa é realizar uma análise da dimensão subjetiva dos hábitos esportivos da população universitária, ou seja, dos variados motivos que levam os estudantes a praticar esporte com maior ou menor intensidade, a abandonar mais ou menos temporalmente a prática e, inclusive, a manifestar o seu desinteresse em tudo aquilo que representa a cultura esportiva considerando diversos fatores, como gênero, idade, nível de prática e gosto pelo esporte.

\section{Metodologia}

\section{Amostra}

Para realizar a seleção de uma amostra tão heterogênea como a formada pela população objeto do nosso estudo, foi necessário utilizar um procedimento de amostras aleatório estratificado por conglomerados. Uma vez definido o tamanho da amostra foram determinadas as proporções considerando o gênero, à formação e ao curso específico, com um erro amostral de $4 \%$. Finalmente, a amostra definitiva ficou composta por 1512 alunos da Universidad de Murcia (Espanha), todos eles de idades compreendidas entre 18 e 45 anos, com idade média de 21,93 anos (61,6\% mulheres e 38,4\% homens). Vale ressaltar que os procedimentos utilizados respeitam as normas internacionais de experimentação com humanos (Declaração de Helsínquia de 1975).

\section{Procedimentos}

Após a revisão dos trabalhos realizados anteriormente sobre questionários de atitudes e motivações que levam à prática de atividades 
físicas ${ }^{8,12,19,20,25}$, elaboramos o questionário dos Interesses nas Atividades Físico-Esportivas e as Motivações que levam à Prática das mesmas (M.I.A.F.D.), com o objetivo de conhecer as opiniões dos universitários sobre a sua relação com a prática de atividades físico-esportivas. Embora esse instrumento conste de duas partes, neste trabalho somente expomos alguns resultados obtidos na segunda parte do questionário, o qual procuramos conhecer as motivações que induzam os universitários a se aproximar das atividades físico-esportivas, diferenciando três grupos: praticantes, não praticantes e ex-praticantes, para o qual se estabeleceram 13-14 motivos em cada um dos subgrupos.

Para recolher os dados utilizamos uma metodologia quantitativa (pesquisa mediante questionário auto-administrado). A pesquisadora principal fez entrevistas pessoais voluntárias, nas quais fazia a apresentação e, se necessário, esclarecia as dúvidas que surgiam, enquanto que para a escolha dos alunos se estabeleceram rendas aleatórias no hall da faculdade universitária selecionada. Os alunos entrevistados tinham que escolher um dos três grupos finalmente formados, em função da relação que tinham mantido com a prática de atividades físico-esportiva.

\section{Estatística}

Para a obtenção dos resultados realizaram-se estatísticas descritivas de todas as variáveis, como também, análises de independência entre variáveis, mediante os testes de $" \chi^{2}$ de Pearson, completados com a análise do resíduo tipificado corrigido.

\section{Resultados}

Inicialmente realizamos uma análise descritiva, enquanto que nas seguintes análises utilizamos como variável dependente os motivos dos universitários para justificar a relação com as atividades físicas e os esportes praticados no tempo livre, e como variáveis independentes o gênero, a idade, o nível de prática e a opinião dos estudantes quanto ao gosto ou não pelo esporte.

\section{Motivações que levam à prática de ativi- dades físico-esportivas}

Na tabela 1, vemos como os 797 praticantes de atividades físico-esportivas se identificam em maior grau com os motivos vinculados à manutenção da forma física $(16,55 \%)$ e aos benefícios que o exercício traz à saúde (16,52\%). Seguem nesta ordem a necessidade de liberar energia e tensões $(15,21 \%)$, como lazer e para passar o tempo $(13,99 \%)$, para melhorar a imagem e o aspecto físico $(11,85 \%)$, para relacionar-se e conhecer gente $(8,66 \%)$, porque gosta de superar-se $(8,10 \%)$ e porque gosta de competir $(5,42 \%)$. Ressaltam, pelo contrário, as baixas porcentagens obtidas alegando motivos tais como agradar a família $(0,12 \%)$, porque está na moda $(0,21 \%)$, pela influência dos professores e treinadores $(0,48 \%)$ e porque seus amigos praticam $(1,04 \%)$.

Os 196 universitários que nunca praticaram nenhuma atividade físico-esportiva durante seu tempo livre baseiam seu comportamento em que tira tempo de estudo $(18,69 \%)$ e na falta de interesse $(18,69 \%)$. Os motivos menos selecionados foram a idade $(0,37 \%)$, a dificuldade de acesso ao esporte $(0,93 \%)$, os motivos de saúde $(1,12 \%)$ e a falta de meios econômicos $(2,06 \%)$. Os 519 alunos que manifestam ter praticado esporte anteriormente, mas que não o fazem atualmente, indicam a falta de tempo livre $(23,5 \%)$ e o nível de exigência dos estudos (20,93\%) como razões para explicar o seu comportamento físico-esportivo. Com menor peso, encontram-se valores baixos de motivações associadas à perda de interesse $(5,22 \%)$, ao desestímulo causado pela desistência da prática dos amigos (4,24\%), à falta de associações esportivas $(3,76 \%)$, à problemas econômicos $(3,62 \%)$, à problemas de saúde $(3,55 \%)$, à falta de informação $(2,09 \%)$, à falta de apoio e estímulo familiar (1,88\%), à idade $(1,53 \%)$ e às más experiências em práticas anteriores $(1,53 \%)$. 
Motivos da prática esportiva de acordo com o nível de competência percebida na idade adulta: um estudo piloto Ana Pavón Lores, Juan Antonio Moreno Murcia, Estélio H. M. Dantas

Tabela 1: Distribuição percentual das motivações que levam à prática de atividades físico-esportivas.

\section{Motivos dos praticantes}

Porque gosto de estar em forma

Pelos benefícios que obtenho para a saúde

Para liberar a energia e as tensões acumuladas durante o dia

Como lazer e para passar o tempo

Para melhorar minha imagem e aspecto físico

Para me relacionar e conhecer gente

Porque gosto de me superar

Porque gosto de competir

Outros motivos

Porque meus amigos praticam

Por influência dos meus professores e treinadores

Porque está na moda

Para agradar a minha família
Frequência Percentagem (\%)

$\begin{array}{cc}556 & 16,55 \\ 555 & 16,52 \\ 511 & 15,21 \\ 470 & 13,99 \\ 398 & 11,85 \\ 291 & 8,66 \\ 272 & 8,10 \\ 182 & 5,42 \\ 62 & 1,85 \\ 35 & 1,04 \\ 16 & 0,48 \\ 7 & 0,21 \\ 4 & 0,12\end{array}$

Motivos dos não praticantes

Frequência Percentagem (\%)

Porque tira tempo de estudo

100

18,69

Por falta de interesse

100

18,69

Por sair cansado do trabalho e/ou aula

$78 \quad 14,58$

Porque nunca tive habilidade para esportes

75

14,02

As instalações estão muito longe do meu domicílio

45

8,41

Falta de instalações e horários adequados

Por falta de apoio e tradição familiar

41

7,66

25

4,67

Porque meus amigos não praticam esporte

Outros motivos

24

4,49

23

4,30

Por falta de meios econômicos

$11 \quad 2,06$

Por motivos de saúde

$6 \quad 1,12$

É difícil ter acesso ao esporte

5

0,93

Por considerar-se muito velho para isso

\section{Motivos de abandono}

2

0,37

Por falta de tempo livre

Frequência Percentagem (\%)

Porque os estudos exigem muito de mim

338

23,50

301

20,93

Porque os horários são incompatíveis com as minhas ocupações

Faltam instalações esportivas adequadas/próximas ao meu domicílio

Porque já não me interessa

Outros motivos

Porque meus amigos deixaram de praticar

Por falta de associações para praticar esporte como lazer

Por problemas econômicos

$52 \quad 3,62$

Por problemas de saúde

Por falta de informação

Porque não existe um apoio e estímulo familiar 
Motivações físico-esportivas de acordo com o gênero

Para $52,7 \%$ da amostra que se declara praticante de atividades físico-esportivas, os principais motivos dos homens são o gosto pela competição $(36,9 \%)$, porque gostam de superar-se $(43,5 \%)$, para relacionar-se e conhecer gente $(43,5 \%)$, porque seus amigos praticam $(6,3 \%)$ e pela influência dos professores e treinadores $(3 \%)$, enquanto que as mulheres não têm interesse por esses motivos. Elas, à diferença dos homens (tabela 2), preferem as atividades físico-esportivas para melhorar a imagem e o aspecto físico $(57,9 \%)$, para liberar a energia e as tensões acumuladas durante o dia $(69,6 \%)$ e pelos benefícios que traz à saúde $(74,1 \%)$.

Do total de 13\% dos estudantes entrevistados que não praticam atividades físico-esportivas, encontramos $8,1 \%$ dos homens que afirmam que é difícil ter acesso ao esporte ( $\mathrm{p}<.05) ; 44,7 \%$ das mulheres que acham que nunca tiveram habilidade para esportes $(\mathrm{p}<.001)$.

Para 34,3\% da amostra que já não pratica atividades físico-esportivas durante o seu tempo livre, os problemas econômicos $(11,2 \%)$, a falta de instalações adequadas ou próximas ao seu domicílio (29,9\%), a exigência dos estudos $(60,1 \%)$, a falta de tempo livre $(67,4 \%)$ e a incompatibilidade de horários com as ocupações diárias $(40,9 \%)$ são motivos vinculados à não prática entre as mulheres, enquanto que os homens são contrários a essa opinião (tabela 2). Além disso, as más experiências em práticas anteriores e a perda de interesse constituem um motivo para $7,2 \%$ e $21,6 \%$ dos homens, enquanto $96,6 \%$ e $87,6 \%$ das mulheres acham o contrário $(\mathrm{p}<.05)$.

Tabela 2: Distribuição percentual, total, $\chi^{2}$ e $p$ valor das motivações que levam à prática de atividades físicas de acordo com o gênero.

Motivações físico-esportivas

Motivos dos praticantes

Porque gosto de competir

Sim, é um motivo de prática.

Não é um motivo de prática

Porque gosto de me superar

Sim, é um motivo de prática

Não é um motivo de prática

Para me relacionar e conhecer gente

Sim, é um motivo de prática

Não é um motivo de prática

Porque meus amigos praticam

Sim, é um motivo de prática

Não é um motivo de prática

Por influência de meus professores

Sim, é um motivo de prática

Não é um motivo de prática

Para melhorar minha imagem /

/ aspecto físico

Sim, é um motivo de prática

Não é um motivo de prática

Para liberar energia e tensões

Sim, é um motivo de prática

Não é um motivo de prática

Pelos benefícios que traz à saúde

Sim, é um motivo de prática

Não é um motivo de prática

\begin{tabular}{|c|c|c|c|c|c|c|}
\hline \multicolumn{2}{|c|}{ Masculino } & \multicolumn{2}{|c|}{ Feminino } & Total & $\chi^{2}$ & p \\
\hline$(\%)$ & Resíduo & $(\%)$ & Resíduo & (\%) & & \\
\hline
\end{tabular}

$\begin{array}{rrrrrrr}36,9 & 10,1 & 6,4 & -10,1 & 23 & 102,654 & .000 \\ 63,1 & -10,1 & 93,6 & 10,1 & 77 & & \\ & & & & & & \\ 43,5 & 5,9 & 23,4 & -5,9 & 34,4 & 35,179 & .000 \\ 56,5 & -5,9 & 76,6 & 5,9 & 65,6 & & \end{array}$

$\begin{array}{rrrrrrr}43,5 & 4,3 & 28,7 & -4,3 & 36,8 & 18,537 & .000 \\ 56,5 & -4,3 & 71,3 & 4,3 & 63,2 & & \end{array}$

$\begin{array}{rrrrrrr}6,3 & 2,7 & 2,2 & -2,7 & 4,4 & 7,498 & .008 \\ 93,8 & -2,7 & 97,8 & 2,7 & 95,6 & & \end{array}$

$\begin{array}{rrrrrrr}3 & 2,2 & , 8 & -2,2 & 2 & 4,674 & .040 \\ 97 & -2,2 & 99,2 & 2,2 & 98 & & \end{array}$

\begin{tabular}{rrrrrrr}
44 & $-3,9$ & 57,9 & 3,9 & 50,3 & 15,278 & .000 \\
56 & 3,9 & 42,1 & $-3,9$ & 49,7 & & \\
& & & & & & \\
60,4 & $-2,7$ & 69,6 & 2,7 & 64,6 & 7,290 & .007 \\
39,6 & 2,7 & 30,4 & $-2,7$ & 35,4 & & \\
& & & & & & \\
66,9 & $-2,2$ & 74,1 & 2,2 & 70,2 & 4,851 & .029 \\
33,1 & 2,2 & 25,9 & $-2,2$ & 29,8 & & \\
\hline
\end{tabular}


Motivos da prática esportiva de acordo com o nível de competência percebida na idade adulta: um estudo piloto Ana Pavón Lores, Juan Antonio Moreno Murcia, Estélio H. M. Dantas

Tabela 2: Distribuição percentual, total, $\chi^{2}$ e $p$ valor das motivações que levam à prática de atividades físicas de acordo com o gênero. (cont.)

Motivações físico-esportivas Masculino

Feminino

Total $\quad \chi^{2} \quad \mathbf{p}$

Motivos dos que nunca praticam

(\%) Resíduo

(\%) Resíduo

(\%)

É difícil ter acesso ao esporte

Sim, é um motivo de abandono

Não é um motivo de abandono

\begin{tabular}{|c|c|c|c|c|}
\hline 8,1 & 2,4 & 1,3 & $-2,4$ & 2,6 \\
\hline 91,9 & $-2,4$ & 98,7 & 2,4 & 97,4 \\
\hline
\end{tabular}

.047

Nunca tive habilidade para esportes

Sim, é um motivo de não prática

$\begin{array}{rrrrrrr}10,8 & -3,8 & 44,7 & 3,8 & 38,3 & 14,553 & .000\end{array}$

Não é um motivo de não prática

89,2

Motivos dos que abandonaram

(\%) Resíduo (\%) Resíduo (\%)

Por problemas econômicos

Sim, é um motivo de abandono

Não é um motivo de abandono

Falta de instalações próximas

Sim, é um motivo de abandono

Não é um motivo de abandono

Os estudos exigem muito

Sim, é um motivo de abandono

Não é um motivo de abandono

Por falta de tempo livre

Sim, é um motivo de abandono

Não é um motivo de abandono

Por incompatibilidade de horários

Sim, é um motivo de abandono

Não é um motivo de abandono

Por más experiências anteriores

Sim, é um motivo de abandono

Não é um motivo de abandono

Porque deixou de me interessar

Sim, é um motivo de abandono

Não é um motivo de abandono

$\begin{array}{rrrrrrr}5,4 & -1,8 & 11,2 & 1,8 & 10 & 3,263 & .048 \\ 94,6 & 1,8 & 88,8 & -1,8 & 90 & & \end{array}$

$\begin{array}{rrrrrrr}21,6 & -1,7 & 29,9 & 1,7 & 28,2 & 2,980 & .049 \\ 78,4 & 1,7 & 70,1 & -1,7 & 71,8 & & \\ & & & & & & \\ 48,6 & -2,2 & 60,1 & 2,2 & 57,7 & 4,692 & .039 \\ 51,4 & 2,2 & 39,9 & -2,2 & 42,3 & & \\ & & & & & & \\ 55 & -2,4 & 67,4 & 2,4 & 64,8 & 5,927 & .019 \\ 45 & 2,4 & 32,6 & -2,4 & 35,2 & & \end{array}$

$\begin{array}{rrrrrrr}23,4 & -3,4 & 40,9 & 3,4 & 37,2 & 11,399 & .001 \\ 76,6 & 3,4 & 59,1 & -3,4 & 62,8 & & \end{array}$

$\begin{array}{rrrrrrr}7,2 & 1,8 & 3,4 & -1,8 & 4,2 & 3,128 & .049 \\ 92,8 & -1,8 & 96,6 & 1,8 & 95,8 & & \end{array}$

\begin{tabular}{rrrrrrr}
21,6 & 2,5 & 12,4 & $-2,5$ & 14,4 & 6,029 & .021 \\
78,4 & $-2,5$ & 87,6 & 2,5 & 85,6 & & \\
\hline
\end{tabular}

Motivações físico-esportivas de acordo com a idade

Entre os praticantes de atividades físico-esportivas, os principais motivos dos menores de 21 anos são liberar a energia e as tensões acumuladas durante o dia $(68,2 \%)$, enquanto que $38,6 \%$ dos maiores de 22 anos rejeitam esta motivação $(\mathrm{p}<.05)$ (tabela 3).

Para os que já não praticam atividades físico-esportivas durante o seu tempo livre, a exigência dos estudos $(61,9 \%)$ e a falta de informação

$(7,7 \%)$ são motivos associados à não prática entre os menores de 21 anos; os maiores de 22 anos que não confirmam esta opinião atingem $47,5 \%$ e $96,6 \%$ respectivamente. No entanto, o fato de que os amigos tenham deixado a prática constitui um motivo para $14,8 \%$ dos maiores de 22 anos, enquanto $90,9 \%$ dos menores de 21 acham o contrário. 
Tabela 3: Distribuição percentual, total, $\chi^{2}$ e $p$ valor das motivações que levam à prática de atividades físicas de acordo com a idade.

Motivações físico-esportivas

Menor de $21 \quad$ Maior de 21 Total $\chi^{2} \quad p$

Motivos dos praticantes

(\%) Resíduo (\%) Resíduo (\%)

Para liberar energia e tensões

Sim, é um motivo de prática

$\begin{array}{lllllll}68,2 & 2 & 61,4 & -2 & 64,6 & 3,976 & .048\end{array}$

Não é um motivo de prática

$\begin{array}{rrrrr}31,8 & -2 & 38,6 & 2 & 35,4\end{array}$

Motivos dos que já praticaram

(\%) Resíduo (\%) Resíduo (\%)

Pela exigência dos estudos

Sim, é um motivo de abandono

Não é um motivo de abandono

$\begin{array}{rrrrrrr}61,9 & 2,2 & 52,5 & -2,2 & 57,7 & 4,626 & .033 \\ 38,1 & -2,2 & 47,5 & 2,2 & 42,3 & & \end{array}$

Por falta de informação

Sim, é um motivo de abandono

Não é um motivo de abandono

$\begin{array}{rrrrrr}7,7 & 2,1 & 3,4 & -2,1 & 5,7 & 4,419\end{array}$

.038

Meus amigos deixaram de praticar

Sim, é um motivo de abandono

Não é um motivo de abandono

$92,3 \quad-2,1 \quad 96,6 \quad 2,1 \quad 94,3$

$\begin{array}{r}9,1 \\ 90,9 \\ \hline\end{array}$

Motivações físico-esportivas de acordo com o nível de prática

Entre os praticantes de atividades físico-esportivas (tabela 4), os principais motivos de prática dos iniciantes são melhorar a imagem e o aspecto físico $(54,6 \%)(\mathrm{p}<.05)$. No entanto, os avançados e os de maior experiência justificam como motivos da prática

que gostam de competir $(28,3 \%$ e 44,3\%, respectivamente $)(\mathrm{p}<.001)$ e que gostam de superar-se $(37,3 \%$ e $54,1 \%$, respectivamente) $(\mathrm{p}<.001)$; no caso dos mais experientes aparece também a influência dos professores e treinadores $(6,6 \%)(\mathrm{p}<.05)$.

Tabela 4: Distribuição percentual, total, $\chi^{2}$ e $p$ valor das motivações que levam à prática de atividades físicas de acordo com o nível de prática.

\begin{tabular}{|c|c|c|c|c|c|c|c|c|c|}
\hline \multirow{2}{*}{$\begin{array}{l}\text { Motivações } \\
\text { Motivos dos praticantes }\end{array}$} & \multicolumn{2}{|c|}{ Iniciante } & \multicolumn{2}{|c|}{ Avançado } & \multicolumn{2}{|c|}{ + Experientes } & \multirow{2}{*}{$\begin{array}{c}\text { Total } \\
(\%)\end{array}$} & \multirow[t]{2}{*}{$\chi^{2}$} & \multirow[t]{2}{*}{ p } \\
\hline & $(\%)$ & Resíduo & $(\%)$ & Resíduo & (\%) & Resíduo & & & \\
\hline \multicolumn{10}{|l|}{ Gosta de competir } \\
\hline Sim, gosto & 12,1 & -6 & 28,3 & 3,6 & 44,3 & 4,1 & 23,1 & 43,038 & .000 \\
\hline Não gosto & 87,9 & 6 & 71,7 & $-3,6$ & 55,7 & $-4,1$ & 76,9 & & \\
\hline \multicolumn{10}{|l|}{ Gosto de me superar } \\
\hline Sim, gosto & 27 & $-3,6$ & 37,3 & 1,7 & 54,1 & 3,4 & 34,5 & 19,688 & .000 \\
\hline Não gosto & 73 & 3,6 & 62,7 & $-1,7$ & 45,9 & $-3,4$ & 65,5 & & \\
\hline \multicolumn{10}{|c|}{ Por causa dos meus professores } \\
\hline Sim, me influenciaram & 1 & $-1,8$ & 2,2 & , 3 & 6,6 & 2,6 & 2 & 8,160 & .017 \\
\hline Não me influenciaram & 99 & 1,8 & 97,8 &,- 3 & 93,4 & $-2,6$ & 98 & & \\
\hline \multicolumn{10}{|c|}{ Para melhorar a minha imagem } \\
\hline Sim & 54,6 & 2 & 48,5 & -1 & 39,3 & $-1,8$ & 50,3 & 5,771 & .046 \\
\hline Não & 45,4 & -2 & 51,5 & 1 & 60,7 & 1,8 & 49,7 & & \\
\hline
\end{tabular}


Motivos da prática esportiva de acordo com o nível de competência percebida na idade adulta: um estudo piloto Ana Pavón Lores, Juan Antonio Moreno Murcia, Estélio H. M. Dantas

Tabela 5: Distribuição percentual, total, $\chi^{2}$ e $p$ valor das motivações que levam à prática de atividades físicas de acordo com o gosto pelo esporte.

Motivações físico-esportivas

Motivos dos praticantes

Porque gosto de competir Sim, é um motivo de prática Não é um motivo de prática

Porque gosto de me superar Sim, é um motivo de prática Não é um motivo de prática

Para me relacionar e conhecer gente Sim, é um motivo de prática Não é um motivo de prática

Como lazer e para passar o tempo

Sim, é um motivo de prática Não é um motivo de prática

Para agradar a minha família

Sim, é um motivo de prática

Não é um motivo de prática

Porque gosto de estar em forma

Sim, é um motivo de prática

Não é um motivo de prática

\section{Motivos dos que nunca praticam}

Faltam instalações e horários

Sim, é um motivo de prática

Não é um motivo de não prática

Nunca tive habilidade para esportes

Sim, é um motivo de prática

Não é um motivo de não prática

Por falta de interesse

Sim, é um motivo de não prática

Não é um motivo de não prática

Motivos dos que abandonaram

Por más experiências anteriores

Sim, é um motivo de abandono

Não é um motivo de abandono

Porque deixou de me interessar

Sim, é um motivo de abandono

Não é um motivo de abandono

Gosta

Não gosta

Total

(\%) Resíduo (\%) Resíduo (\%)

$\begin{array}{rrrrrrr}23,6 & 2,5 & 0 & -2,5 & 23 & 6,142 & .007 \\ 76,4 & -2,5 & 100 & 2,5 & 77 & & \end{array}$

$\begin{array}{rrrrrrr}35,1 & 2,8 & 5 & -2,8 & 34,4 & 7,854 & .003 \\ 64,9 & -2,8 & 95 & 2,8 & 65,6 & & \end{array}$

$\begin{array}{lllllll}37,5 & 2,5 & 10 & -2,5 & 36,8 & 6,332 & .010\end{array}$

$\begin{array}{lllll}62,5 & -2,5 & 90 & 2,5 & 63,2\end{array}$

$\begin{array}{rrrrrrr}59,9 & 1,8 & 40 & -1,8 & 59,4 & 3,209 & .049 \\ 40,1 & -1,8 & 60 & 1,8 & 40,6 & & \end{array}$

$\begin{array}{rrrrrrr}, 4 & -2,9 & 5 & 2,9 & , 5 & 8,238 & .049 \\ 99,6 & 2,9 & 95 & -2,9 & 99,5 & & \end{array}$

$\begin{array}{rrrrrrr}70,8 & 2 & 50 & -2 & 70,3 & 4,045 & .048\end{array}$

$\begin{array}{lllll}29,2 & -2 & 50 & 2 & 29,7\end{array}$

(\%) Resíduo (\%) Resíduo (\%)

\begin{tabular}{rrrrrrr}
26,9 & 2,6 & 11,7 & $-2,6$ & 20,9 & 6,531 & .012 \\
73,1 & $-2,6$ & 88,3 & 2,6 & 79,1 & & \\
& & & & & & \\
26,9 & $-4,1$ & 55,8 & 4,1 & 38,3 & 16,590 & .000 \\
73,1 & 4,1 & 44,2 & $-4,1$ & 61,7 & & \\
& & & & & & \\
43,7 & $-2,5$ & 62,3 & 2,5 & 51 & 6,500 & .013 \\
56,3 & 2,5 & 37,7 & $-2,5$ & 49 & & \\
\hline
\end{tabular}

(\%) Resíduo (\%) Resíduo (\%)

\begin{tabular}{rrrrrrr}
3,4 & $-2,5$ & 10,3 & 2,5 & 4,2 & 6,074 & .026 \\
96,6 & 2,5 & 89,7 & $-2,5$ & 95,8 & & \\
& & & & & & \\
11,6 & -5 & 36,2 & 5 & 14,4 & 25,294 & .000 \\
88,4 & 5 & 63,8 & -5 & 85,6 & & \\
\hline
\end{tabular}




\section{Motivações físico-esportivas de acordo com o gosto pelo esporte}

De um total de $52,7 \%$ da amostra praticante de atividades físico-esportivas, os alunos que gostam de esporte escolhem como motivos de prática: o gosto pela competição $(23,6 \%)$ $(\mathrm{p}<.01)$, porque gostam de superar-se $(35,1 \%)$ $(\mathrm{p}<.01)$, para relacionar-se e conhecer gente $(37,5 \%)(\mathrm{p}<.01)$, como lazer e para passar o tempo $(59,9 \%)(\mathrm{p}<.05)$ e porque gostam de estar em forma $(70,8 \%)(p<.05)$. No entanto, para os alunos que não gostam de praticar esporte $o$ principal motivo de prática é agradar a família $(5 \%)(\mathrm{p}<.05)($ tabela 5$)$.

Além disso, os entrevistados que gostam de esporte, que nunca praticaram nenhuma atividade físico-esportiva em seu tempo livre, baseiam a falta de motivação na carência de instalações e horários adequados $(26,9 \%)(\mathrm{p}<.05)$, enquanto os entrevistados que não gostam de esporte, a falta de motivação está no fato de nunca ter tido habilidade para esportes $(55,8 \%)(\mathrm{p}<.001)$ e na falta de interesse $(62,3 \%)(\mathrm{p}<.05)$.

Por último, os universitários que não gostam de esporte e que já o praticaram anteriormente, mas não o fazem atualmente, justificam a decisão nas más experiências em práticas anteriores $(10,3 \%)(\mathrm{p}<.05)$ e na perda de interesse $(36,2 \%)$ $(\mathrm{p}<.001)$. Opinião totalmente contrária à manifestada por aqueles alunos universitários que gostam de esporte $(96,6 \%$ e $88,4 \%$, respectivamente) (tabela 5).

\section{Discussão}

Levando em conta que as motivações são conceitos dinâmicos que variam em função do tempo de prática e que raramente se concretizam em um só motivo ${ }^{16,6}$, pretendemos aprofundar a análise dos hábitos físico-esportivos da população universitária com o objetivo de concretizar quais são os motivos que vão determinar entre os estudantes universitários o interesse pela prática de esportes ou atividades físicas ou o abandono mais ou menos temporário da prática e, inclusive, a falta de interesse e relação a tudo o que representa o entorno físico-esportivo.

Desta forma, os praticantes de atividades físico-esportivas vão explicar a participação nesse tipo de atividades, por motivos vinculados à manutenção da forma física e aos benefícios que o exercício traz à saúde. Outros motivos são a necessidade de liberar energia e tensões, como lazer e para passar o tempo, para melhorar a imagem e o aspecto físico, para relacionar-se e conhecer gente, porque gostam de superar-se e porque gostam de competir. Ressaltam, pelo contrário, as baixas porcentagens obtidas alegando motivos como agradar a família, porque está na moda, pela influência dos professores e treinadores e porque os amigos o fazem.

Entre a população universitária encontramos diversos estudos que abalam esses resultados ${ }^{40,14,33}$, indicando a preferência dos estudantes pelo lazer, a saúde e a forma física, e o bem-estar geral. Enquanto outros autores ${ }^{25,29}$ indicam como grandes motivos para a prática de esportes, aqueles com relação à saúde e à interação social. De fato, quando os estudos focalizam a população em geral, os motivos que levam as pessoas à prática de esportes estão vinculados à "fazer exercício físico", "por lazer", "porque gosta de esportes" e para "manter e melhorar a saúde", motivos que prevalecem sobre as motivações de caráter competitivo ${ }^{4,11}$.

Quando Cervelló 6 fala do fenômeno do abandono esportivo estabelece que deve ser considerado como um fato contínuo, que vai desde pessoas que deixam de praticar um esporte em particular, mas passam a praticar outro, ou o mesmo esporte, mas em diferente nível de intensidade, até aquelas que abandonam definitivamente o esporte. A partir dessa idéia e para simplificar 
a análise, foi necessário diferenciar os universitários que nunca praticaram nenhuma atividade físico-esportiva em seu tempo livre dos que não o estão fazendo atualmente, seja de forma temporal ou definitiva, mas que antes a exerciam. Desta forma, os primeiros baseiam seu comportamento em que a prática físico-esportiva tira tempo de estudo e na falta de interesse ${ }^{5,10,26,8,4}$. Alegam também, justificando sua atitude, que saem cansados do trabalho e/ou aula, que nunca tiveram habilidade para esportes, que as instalações estão muito longe, que faltam horários e instalações adequados, que falta apoio e tradição familiar e que os amigos não praticam esportes. Os motivos selecionados em menor grau foram a idade, a dificuldade de acesso ao esporte, os motivos de saúde e a falta de meios econômicos. Tal como podemos observar em vários trabalhos $^{41,12}$, aqueles que não praticam esporte se destacam entre os que indicam que o praticariam por motivos alheios ao próprio esporte, tais como econômicos e profissionais. Por outro lado, os alunos que manifestam já ter praticado algum esporte, mas não o fazem atualmente, indicam a falta de tempo livre e o nível de exigência dos estudos como razões para explicar o seu comportamento físico-esportivo ${ }^{18,40,14,26}$. Outros motivos são a incompatibilidade de horários e a falta de instalações esportivas adequadas ou próximas ao seu domicílio ${ }^{7}$. Em ordem decrescente aparecem motivações associadas à perda de interesse, ao desestímulo causado pela desistência da prática dos amigos, à falta de associações esportivas, aos problemas econômicos, aos problemas de saúde, à falta de informação, à falta de apoio e estímulo familiar, à idade, e às más experiências em práticas anteriores.

Resultados estes que não são muito díspares dos encontrados em outras pesquisas ${ }^{4,9,5}$, nas quais se menciona como motivos que levaram a população espanhola ao abandono da prática de atividades esportivas: "por falta de tempo", "por sair cansado do trabalho", "pela saúde (lesões etc.)" e "por preguiça e desânimo". Destacam o estudo de Ruiz e García ${ }^{26}$, no qual os universitários almerienses selecionam a falta de tempo devido à exigência do trabalho ou estudo, seguido da preguiça ou desânimo, o horário incompatível com suas obrigações, gostar mais de outras coisas, a não prática dos seus amigos e a distância de seu domicílio, como motivos que explicam o abandono da prática.

Paralela e inevitavelmente a influência dos estereótipos no ensino da Educação Física se traduz numa série de diferenças, tanto de interesses e motivações quanto no grau de participação em certas atividades físicas, em função do gênero do aluno, tal como foi detectado em numerosas pesquisas ${ }^{18,7,21,34,35,20,29}$. Desta forma, o gênero dos alunos vai influenciar na percepção da atividade física e da Educação Física, como também no seu envolvimento com o esporte e as razões que o levam à praticá-lo. Não obstante, devemos entender que a participação de mulheres e homens em atividades físicas e esportivas não é somente quantitativa, como também, varia dependendo de fatores como o tipo de esporte, as motivações da prática, a facilidade ou dificuldade de acesso e a disposição e distribuição do tempo livre ${ }^{35,38,39}$.

Realmente relevante é que o gênero é, junto com a idade, o principal elemento diferenciador dos motivos da prática esportiva ${ }^{2,27}$. Para os alunos da Universidad de Murcia que se declaram praticantes de atividades físico-esportivas, os principais motivos dos homens são porque gostam de superar-se, para relacionar-se e conhecer gente, porque gostam de competir, porque os amigos praticam e pela influência dos professores e treinadores. Não obstante, as praticantes preferem as atividades físico-esportivas pelos benefícios que traz à saúde, para liberar a energia e as tensões acumuladas durante o dia e para melhorar a imagem e o aspecto físico. $\mathrm{Ou}$ seja, os homens valorizam mais aqueles aspectos vinculados à capacidade pessoal, à competição, ao hedonismo e às relações sociais, enquanto as motivações que levam as mulheres à pratica de atividades físico-esportivas estão mais relacionadas com a forma física, a imagem pessoal e a 
saúde, tal como tínhamos antecipado ao conceber as hipóteses iniciais. Estes resultados coincidem com os encontrados em numerosas pesquisas $^{22,37,1,41}$, nas quais os homens eram identificados com motivos competitivos, de relação social e de lazer, e as mulheres com a saúde, os fins terapêuticos e a estética corporal.

Dos estudantes entrevistados que não praticam atividades físico-esportivas em seu tempo livre, os homens afirmam que é difícil ter acesso ao esporte, enquanto as mulheres acham que nunca tiveram habilidade para esportes. Além do mais, para os alunos que já não praticam atividades físico-esportivas durante o seu tempo livre, a falta de tempo livre, a exigência dos estudos, a incompatibilidade de horários com as ocupações diárias, a falta de instalações adequadas ou próximas ao seu domicílio e os problemas econômicos são motivos vinculados ao abandono entre o gênero feminino. Enquanto para os homens são a perda de interesse e as más experiências em práticas anteriores que constituem as motivações básicas que levam ao abandono. Estes resultados diferem dos encontrados por Salguero et $a l^{28}$, em que se detectam escassas diferenças entre homens e mulheres nas causas de abandono. Assim, a habilidade percebida tinha uma maior contribuição entre as mulheres, enquanto a falta de interesse se considerava de maior importância entre os homens.

Como já mencionamos, a análise levando em conta a idade é fundamental, já que constitui um grande elemento diferenciador no estudo dos motivos de prática físico-esportiva ${ }^{3,39}$. De fato, a idade média de abandono oscila em torno aos $18 \operatorname{anos}^{28}$, que é quando se iniciam os estudos universitários, com o grande salto qualitativo e quantitativo que isto implica, o que junto com a falta de coordenação entre a estrutura esportiva e a acadêmica vai favorecer o abandono do esporte. Encontramos numerosos estudos dedicados a explicar as motivações que guiam os praticantes em função da idade. Desta forma, alguns trabalhos ${ }^{15}$ mencionam que os mais jovens estão mais motivados por fatores externos e motivos extrínsecos, outros citam $^{33,40}$ que o componente divertido do esporte e da atividade física é a principal motivação entre os jovens, diminuindo à medida que vão amadurecendo e aumentando a necessidade de ser eficaz, se esforçar e ter êxito, enquanto, outros estudos ${ }^{18,22}$ estabelecem que em idades menores há um maior interesse em relação ao rendimento, ao lazer e à relação social, diminuindo sua importância à medida que aumentam a idade e os motivos vinculados à saúde. Entre os praticantes de atividades físico-esportivas, os principais motivos dos menores de 21 anos são em liberar a energia e as tensões acumuladas durante o dia, enquanto os maiores de 22 anos rejeitam esta motivação. Para os que já não praticam atividades físico-esportivas durante seu tempo livre, a exigência dos estudos e a falta de informação são motivos associados ao abandono entre os menores de 21 anos. No entanto, o fato de que os amigos tenham deixado a prática constitui um motivo importante para os maiores de 22 anos.

Nas motivações que levam à prática de atividades físico-esportivas, evidencia-se a relação estabelecida entre o nível de prática e o tipo de esporte praticado. Desta forma, os alunos avançados e mais experientes justificam como motivos da prática o fato de que gostam de superar-se e de competir; os que gostam de competir, acrescentam a influência dos professores e treinadores, tudo em evidente relação com o esporte de competição. Ficam para os iniciantes, todos aqueles motivos vinculados à melhorar a imagem pessoal e o aspecto físico, predominantes entre as atividades saudáveis e lúdico-recreativas próprias do esporte para todos. Isto está em consonância com os resultados obtidos em outras pesquisas ${ }^{23}$, que parecem indicar que quanto maior é o nível de prática maior é o interesse demonstrado em relação aos valores competitivos e sociais do esporte, enquanto nos níveis inferiores parecem predominar as motivações próximas à preocupação com a imagem 
pessoal e ao conceito do esporte-saúde como elemento indispensável para uma melhor qualidade de vida.

Se levarmos em conta o gosto manifestado pela amostra em relação com o esporte e as atividades físico-esportivas, os praticantes que gostam de esporte mencionam como motivos de prática que gostam de estar em forma, o lazer e passar o tempo, para relacionar-se e conhecer gente, prazer de superar-se e o prazer de competir. No entanto, para os alunos que não gostam de esporte os principais motivos de prática são agradar a família, evidenciando a importância do apoio social na adoção e manutenção de padrões de exercício ${ }^{17,13}$. Entre os estudantes que gostam de esportes apesar de nunca terem praticado nenhuma atividade físico-esportiva em seu tempo livre, a falta de motivação se baseia na carência de instalações e horários adequados, enquanto para os entrevistados que não gostam de esportes, a ausência de motivação deve ter fundamento no fato de nunca ter tido habilidade e na falta de interesse. O grupo de universitários que não gosta de esporte e também não tem prática físico-esportiva no momento, justifica decisão pela perda de interesse e pelas más experiências acumuladas em práticas anteriores.

Definitivamente, as motivações são conceitos dinâmicos, já que as necessidades pessoais mudam com o passar do tempo. Por isso é fundamental conhecer o estabelecimento diferencial das motivações para cada momento e a sua evolução ao longo do tempo se nossa pretensão é fomentar a idéia da mudança de atividade esportiva $v s$. a opção de abandono, na busca de um estilo de vida ativo durante a idade adulta.

Ressalta-se no entanto, que o presente estudo é uma pesquisa preliminar, e que oportunamente o questionário apresentado deverá ser validado através de métodos estatísticos apropriados (análise factorial exploratória e confirmatória).

\section{Agradecimentos}

A informação contida neste artigo faz parte da Tese de doutorado apresentada por Ana Pavón Lores, no ano de 2004: "Motivaciones e intereses de los universitarios murcianos hacia la práctica físico-deportiva" (Motivações e interesses dos universitários murcianos que levam à prática físico-esportiva), dirigida pelo Dr. Juan Antonio Moreno da Universidad de Murcia (Espanha).

\section{Correspondência}

Juan Antonio Moreno Murcia

Facultad de Ciencias del Deporte

Universidad de Murcia, Parque Almansa

30730 San Javier, Murcia, Spain

Tel.: 968193358 ext. 21

E-mail: morenomu@um.es 


\section{Referências}

1. Blasco, T.; Capdevila, Ll.; Pintanel, M.; Valiente, L.; Cruz, J. (1996). Evolución de los patrones de actividad física en estudiantes universitarios. Revista de Psicología del Deporte, 9-10: 51-63.

2. Bungum, T.; Dowda, M.; Weston, A.; Trost, S. G.; Pate, R. (2000). Correlates of physical activity in male and female youth. Pediatric Exercise Science, 12(1): 71-79.

3. Campbell, P. G.; MacAuley, D.; McCrum, E.; Evans, A. (2001). Age differences in the motivating factors for exercise. Journal of Sport \& Exercise Psychology, 23(3): 45-57.

4. Centro de Investigaciones Sociológicas (1997). Informe sobre tiempo libre y deportes. Noviembre 1997. Estudio no 2.266 .

5. Centro de Investigaciones Sociológicas (2000). Informe sobre la juventud española. Octubre-Noviembre 1999. Estudio no 2.370.

6. Cervelló, E. M. (2000). Una explicación de la motivación deportiva y el abandono desde la perspectiva de la Teoría de Metas: Propuestas para favorecer la adherencia a la práctica deportiva. Primer Congreso Hispano-Portugués de Psicología. Santiago de Compostela.

7. Fan, L.; Gu, M.; Wang, H.; Yu, X.; Lu, M.; Wang, X. N. (2000). A nationwide survey on students' physical activities after school. Sport Science, 20(2): 7-11.

8. García Ferrando, M. (2001). Los españoles y el deporte: Prácticas y comportamientos en la última década del siglo XX. Encuesta sobre los hábitos deportivos de los españoles, 2000. Madrid: C. S. D. y Ministerio de Educación, Cultura y Deporte.

9. García Ferrando, M. (2005). Encuesta sobre hábitos deportivos de los españoles. Avance de resultados: C. S. D. y C. I. S.

10. Gómez, M.; Ruiz, F.; García, M. E.; Piéron, M. (2003a). Evolución de los hábitos deportivos de los estudiantes de la Universidad de Almería. Actas del IX Congreso Nacional de Psicología de la Actividad Física y el Deporte "Perspectiva Latina". León: Asociación de Psicología del Deporte, 79-84.

11. Gómez, M.; Ruiz, F; García, M. E.; Piéron, M. (2003b). Motivos aludidos, por el alumnado de Educación Secundaria Post-Obligatoria, para practicar actividades físico-deportivas: Un estudio longitudinal. Actas del II Congreso Mundial de Ciencias de la Actividad Física y del Deporte: Deporte y Calidad de vida. Area 6. Enseñanza de la Educación Física. Granada: Facultad de Ciencias de la Actividad Física y el Deporte, 118-123.

12. Gutiérrez, M.; González-Herrero, E. (1995). Motivos por los que los jóvenes practican actividad física y deportiva: análisis de una muestra de estudiantes universitarios. Aplicacions $i$ fonaments de les activitats físico-esportives. Lleida: INEFC, 363-374.

13. Gutiérrez, M. (2000). Actividad física, estilos de vida y calidad de vida. Revista de Educación Fisica, 77: 5-14.

14. Llopis, D.; Llopis, R. (1999). Motivación y práctica deportiva. In: Nieto, G.; Garcés de Los Fayos, E. J. (Eds.). Psicología de la Actividad Fúsica y el Deporte, Tomo I. Murcia: Sociedad Murciana de Psicología de la Actividad Física y el Deporte, 127-134.

15. López, C.; Márquez, S. (2001). Motivación en jóvenes practicantes de lucha leonesa. Revista de Psicología del Deporte, 10, 1: 9-22.

16. Masachs, M.; Puente, M.; Blasco, T. (1994). Evolución de los motivos para participar en programas de ejercicio físico. Revista de Psicología del Deporte, 5: 71-80.

17. Miguel Salgado-Araujo, J. L. (1998). Revisión de la literatura actual sobre la continuidad del cambio de conducta em relación a la actividad física. Apunts: Educación Física y Desportes, 56: 66-77. 
18. Moreno, J. A.; Gutiérrez, M. (1998b). Intereses, actitudes y motivación hacia la práctica terrestre y acuática de los españoles. In: Moreno, J. A.; Rodríguez, P. L.; Ruiz, F. (Eds.). Actividades acuáticas: Ámbitos de aplicación. Murcia: Universidad de Murcia, 185-204.

19. Moreno, J. A.; Rodríguez, P. L.; Gutiérrez, M. (1996). Actitudes hacia la Educación Física: Elaboración de un instrumento de medida. Actas del III Congreso Nacional de Educación Física de Facultades de Educación y XIV de Escuelas Universitarias de Magisterio. Guadalajara: Universidad de Alcalá, 507-516.

20. Pavón, A. (2001). Intereses y actitudes hacia la práctica físico-deportiva en la etapa universitaria. Trabajo presentado para la obtención del DEA. Murcia: Universidad de Murcia.

21. Pavón, A. (2004). Motivaciones e intereses de los universitarios murcianos hacia la práctica físicodeportiva. Tesis doctoral. Dir. Dr. Juan Antonio Moreno. Murcia: Universidad de Murcia.

22. Pavón, A.; Moreno, J. A.; Gutiérrez, M.; Sicilia, A. (2003a). La práctica físico-deportiva en la Universidad. Revista de Psicología del Deporte, 12, 1: 39-54.

23. Pavón, A.; Moreno, J. A.; Gutiérrez, M.; Sicilia, A. (2003b). Intereses y motivaciones de los universitarios: diferencias en función del nivel de práctica. Cuadernos de Psicología del Deporte, 3, 1: : 33-43.

24. Piéron, M.; Telama, R.; Almond, L.; Carreiro da Costa, F. (1999). Estilo de vida de jóvenes europeos: Un estudio comparativo. Revista de Educación Física, 76: 5-13.

25. Reyes, S.; Garcés, E. (1999). ¿Por qué las personas hacen deporte?. Un análisis descriptivo en una población de estudiantes universitarios. In: Nieto, G.; Garcés de Los Fayos, E. J. (Eds.). Psicología de la Actividad Física y el Deporte. Murcia: Sociedad Murciana de Psicología de la Actividad Física y el Deporte, 121-126.
26. Ruiz, F.; García, E. (2002). El abandono y la ausencia de práctica de actividad físico-deportiva de tiempo libre del alumnado de la universidad de Almería. Un estudio longitudinal. Revista Digital Lecturas: Educación Física y Deportes, 8, 47. http://www.efdeportes.com/efd47/aband.htm

27. Ruiz, F.; García, E.; Hernández, A. I. (2001). El interés por la práctica de actividad físico-deportiva de tiempo libre del alumnado de la Universidad de Almería. Un estudio longitudinal. Apunts: Educación Física y Deportes, 63: 86-92.

28. Salguero, A.; Tuero, C.; Márquez, S. (2003). Adaptación española del Cuestionario de Causas de Abandono en la Práctica Deportiva: validación y diferencias de género en jóvenes nadadores. Revista Digital Lecturas: Educación Física y Deportes, 8 (56).

http://www.efdeportes.com/efd56/aband.htm

29. Sánchez, A.; García, F.; Landabaso, V.; Nicolás, L. (1998). Participación en actividad física de una muestra universitaria a partir del modelo de las etapas de cambio en el ejercicio físico: un estudio piloto. Revista de Psicología del Deporte, 7(2): 233-245.

30. Sánchez-Barrera, M.; Pérez, M.; Godoy, J. (1995). Patrones de actividad física de una muestra española. Revista de Psicología del Deporte, 7-8: 51-71.

31. Sánchez, E.; García, J. M. A. (2001). Análisis de las motivaciones para la participación en la comunidad. Papers: Revista de Sociología, 63/64: 171-189.

32. Savage, M. P. (1998). University students' motivation for participation in a basic instruction program. College Student Journal, 32, 1: 58-65.

33. Segura, J.; Cebriá, J.; Casas, O.; Corbella, S.; Crusat, M.; Escanilla, A.; Grau, G.; Sanromá, M. (1999). Hábitos de actividad física en estudiantes universitarios. In: Nieto, G.; Garcés de Los Fayos, E. J. (Eds.). Psicología de la Actividad Física y el Deporte. Murcia: Sociedad Murciana de Psicología de la Actividad Física y el Deporte, 203-213. 
34. Scraton, S.; Flintoff, A. (2002). Gender and sport: $A$ reader. London: Routledge.

35. Sicilia, A. (2002). Desigualdad y género en la Educación Física escolar. Actas del III Congreso Internacional de Educación Física. Jeréz de la Frontera: FETE-UGT, 679-697.

36. Taylor, W. C.; Blair, S. N.; Cummings, S. S.; Wun, C. C.; Malina, R. M. (1999). Childhood and adolescent physical activity patterns and adult physical activity. Medicine and Science in Sports and Exercise, 31(1): 118-123.

37. Torre, E.; Cárdenas, D.; García, E. (2001). La motivación en la práctica físico-deportiva. Revista Digital Lecturas: Educación Física y Deportes, 7, 39. http://www.efdeportes.com/efd39/motiv.htm
38. Vázquez, B. (2001). La cultura física y las diferencias de género en el umbral del siglo XXI. In: Devís, J. (Ed.). La Educación Física, el Deporte y la Salud en el siglo XXI. Alcoy: Marfil, 213-226.

39. Wang, C. K. J.; Biddle, S. J. H. (2001). Young people's motivational profiles in physical activity: A Cluster Analysis. Journal of Sport \& Exercise Psychology, 23 (1).

40. Weinberg, R.; Gould, D. (1996). Fundamentos de psicología del deporte y el ejercicio físico. Barcelona: Ariel Psicología.

41. White, S. A. (1995). The perceived purposes of sport among male and female intercollegiate and recreational sport participants. International Journal of Sport Psychology, 26: 490-502. 